Celal Bayar University Journal of Science

\title{
Noise Analysis of Air Disc Brake Systems Using Wavelet Synchrosqueezed Transform
}

\author{
Zeynep Ertekin ${ }^{1 *}$, Nalan Özkurt ${ }^{1}$ \\ ${ }^{1}$ Yaşar University, Selçuk Yaşar Campus, 35100 Bornova, İzmir, Turkey \\ * zeynep.ertekin@yasar.edu.tr
}

Received: 5 February 2019

Accepted: 12 December 2019

DOI: $10.18466 /$ cbayarfbe. 522686

\begin{abstract}
Recently, signal processing methods are shown to be successful while diagnosing faults in mechanical systems, using noise or vibration data. In this study, two different faulty air disc brakes; noisy and less noisy ones are investigated using Wavelet Synchrosqueezed Transform on audio recordings. The difference between two types are shown in scalogram and also verified by a quantitative measure of entropy. The audio recording has been carried out by using two identical microphones sited on the brakes via data acquisition unit at a sampling rate of $20 \mathrm{kHz}, 16$-bit resolution and these data are analyzed in MATLAB software. The average of the entropy values of faulty and non-faulty brakes were found to be 0.98 and 0.65 , respectively. Therefore, it has been concluded that, the entropy could be used as a distinguishing tool to discriminate the faults.
\end{abstract}

Keywords: Air Disc Brake, Entropy, Noise analysis, Sound Processing, Wavelet Synchro Squeezed Transform.

\section{Introduction}

Fast and safe transportation has an important role in our business and social life. Ensuring safety both in passenger cars and heavy vehicles is one of the engineering problems yet more to tighten the measures taken for safety. Due to its' complex nature, brake faults are hard to notice, every time driver or controller suspects an error, it has to be removed and examined, which refers to waste of workforce time and even money. Any error or even doubt on the brake system must be taken into consideration seriously since it would affect not only a single person's safety but also the rest of the traffic (passengers, commercial/personal vehicles, animals etc.). Hence, any problem in brakes must be investigated thoroughly until satisfactory answers/solutions are obtained. Right in this point, significance of automatic and semi-automatic fault detection systems are highlighted by both manufacturers and engineers. Brake is the most indispensable safety measure of a vehicle which is quite significant in this context where unexpected noise and vibration from the brake provide information about its status, being faulty or not. Such noise and vibration problems are encountered mainly in highway, railway, airway and off-road vehicles, large-scale work machines, oil drilling rigs and mine lifting systems [1]. Due to related studies in literature and feedbacks from manufacturers, noise and accompanying vibration mostly occur as a result of the material of the intermediate parts in the brake, wear over time, or the dimensional errors in the design stage.

In a study conducted by Rhee et al. the sounds and vibrations received from the brakes are divided into two main categories: low frequency $(100-1000 \mathrm{~Hz})$ and high frequency (1000-18000 Hz) [2]. Low frequency sound and vibration fault occurs mostly as a consequence of rotor thickness, thermal effects or too much contamination of the frictional material during braking to the rotor surface whereas high frequency faults are thought to be caused by continuous rotation and bending of the brake pads [1]. In the literature, there exist some case-studies explained by Hammer Effect or Mindlin's Theory, nevertheless with developing technology Noise Vibration and Harshness (NVH) issues are getting more and more complicated due to thriving mechanism behind braking [2]. Even though such systems are mechanical, investigating NVH problems are frequently done by signal processing techniques and not only in brakes but also in various machinery systems since 1900s [1-4].

In spite of the fact that Joseph Fourier is known as the head of signal processing introducing Fourier Transform 
(FT), this approach sometimes might be inadequate in time varying systems. Mechanical deformation under non-stationary conditions could only be thoroughly observed in time-varying characteristics however can hardly be recognized through time domain or FT analysis. Chen et al. showed the potential gear fault by presenting time-frequency reassignment technique to the wavelet scalogram where experimental results point out that real-world gear fault is effectively identified [3].

He et al. showed that Short Time Fourier Transform (STFT), Synchrosqueezed Transform (SST) and TimeReassigned Synchrosqueezed Transform (TSST) methods can be effectively used in the analysis of mechanical systems both theoretically and experimentally by using noise and vibration data [4]. Reassigned wavelet scalogram approach has been used by Chen and Feng again in order to extract fault feature from wind turbine planetary gearbox vibration signals [5]. A new method is proposed to reduce noise on rolling element bearing (REB) using synchronous averaging reassigned wavelet scalogram (SARWS) where both simulated and real signals are analyzed [6]. Peng et al. put forward new methods to reduce border distortion in scalogram which improves estimating modal parameters of two degree of freedom systems [7]. Same researchers used wavelet scalogram and reassigned wavelet scalogram in order to analyze vibration issue for three different kind of faults; rotor to stator rub impact, oil whirl and coupling misalignment. Reassigned scalogram exhibited the best results where both simulation signal and experimental data have been used [8]. SST method is not only applied in mechanical systems but also used in different areas such as seismic processing in geophysics [9-10], in radar applications target imaging [11] and in AM-FM images' [12] demodulation.

The fault investigated in this study is reported by drivers as; there exists an unwanted noise when the brake is hit. Brake, as expressed in the following chapter is a quite complex and a multicomponent mechanism. Just as Rhee and his friends put forward [2], these type of noise occur due to wear of some elements or optimization mistakes in design processes. In this study, sound data collected from air disc brakes are evaluated using Synchrosqueezed Wavelet Scalogram. The sound data were collected from a noisy and a less noisy air disc brakes, which only the experienced audience could distinguish, via microphone, on the vehicle in Ege Fren A.Ş. laboratories. 150 seconds braking audio arising from faulty air disc brakes has been recorded and transferred to computer via a data acquisition board. Same data has been analyzed in a previous study by using Complex Continuous Wavelet Transform [13] Novelly, in this study differences between less noisy and noisy brakes are represented by Synchrosqueezed Wavelet Scalogram approach. Entropies of the ridges of the scalogram matrix also prove the difference in between them. With this new approach, a rigorous method for discriminating faulty brakes from the normal ones has been proposed.

\section{Materials and Methods 2.1. Air Disc Brake}

Brakes are mainly classified into three with respect to their working principles; frictional, pumping and electromagnetic. Sometimes, a braking system could be using a combination of these mechanisms. Mechanic, hydraulic, pneumatic or electromagnetic force is used in order to slow down or stop a vehicle in motion. In early future, brake-by-wire systems are going to substitute conventional hydraulic brakes possibly [14]. Air disc brakes are often used in heavy vehicles and passenger cars which is at issue in this work, shown in figure below.

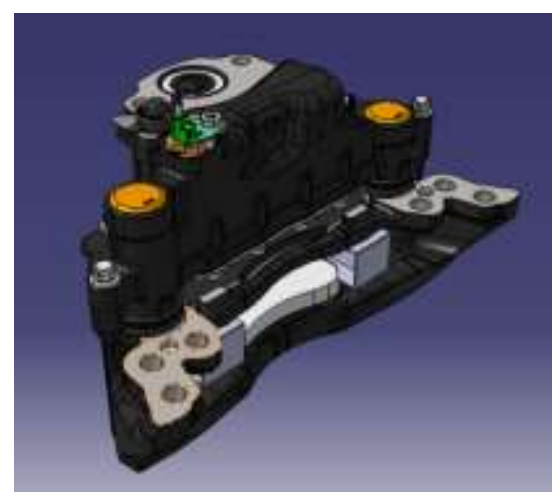

Figure 1. Air Disc Brake.

The force generated by drivers' hit on pedal is transmitted to brake discs pneumatically, based upon Pascal's law. Lining, brake shoe, discs and pistons are the most important components of this complex system. Lining is a half-moon shaped structure that stands on the wheel shaft. It is a material with slow abrasion, water and oil resistant even has a great coefficient of friction in hot and cold weather. The friction surface of the pads is the rotor surface and the braking effect occurs on the component [15]. The force applied to the pneumatic piston in the brake structure is increased by means of the shaft assembly. The power that is released by the friction of the brake pads and sensed on the rotor is transferred to the carrier via the end of the pads firmly connected to the axle.

\subsection{Continuous Wavelet and Synchrosqueezed Wavelet Transform}

Wavelets are actually a family which satisfies certain mathematical criteria where a mother-wavelet could be scaled and translated [16]. Coefficients of Continuous Wavelet Transform (CWT) of a finite energy signal $s(t)$ $\epsilon \mathrm{L}^{2}(\mathbb{R})$ is defined; 


$$
\mathrm{W}_{\mathrm{s}}(\mathrm{a}, \mathrm{b} ; \psi) \triangleq \int_{-\infty}^{\infty} s(t) \psi_{a, b}^{*}(t) d t
$$

where $\mathrm{a}$ and $\mathrm{b}$ denotes scale and translation parameters and "*" represents the complex conjugate.

$$
\psi_{\mathrm{a}, \mathrm{b}}(\mathrm{t}) \triangleq \frac{1}{a^{1 / 2}} \psi\left(\frac{t-b}{a}\right), \mathrm{a} \in \mathbb{R}^{+}, \mathrm{b} \in \mathbb{R}
$$

$\psi($.$) is the mother wavelet which must satisfy$ admissibility criteria.

$$
0<c_{\psi} \triangleq \int_{0}^{\infty}|\widehat{\psi}(\omega)|^{2} \frac{d \omega}{|\omega|}<\infty
$$

$\widehat{\psi}(\omega)$ shows the Fourier Transform of mother wavelet. Scalogram $\mathrm{P}_{\mathrm{s}}(\mathrm{a}, \mathrm{b} ; \psi)$, exhibits local time-frequency energy concentration using wavelet coefficients, and defined as:

$$
P_{s}(a, b ; \psi) \triangleq \mid W_{s}\left(a, b ;\left.\psi\right|^{2}\right.
$$

Basically, it shows signal energy distribution on timefrequency plane.

The Wavelet Synchro-Squeezed Transform (WSST) is a time-frequency technique that reallocates the signal energy in frequency. The reassignment recompenses for the spreading effects caused by the mother wavelet [17]. Contrasting other time-frequency reassignment approaches, synchro-squeezing reassigns the energy just in the frequency direction, which protects the time resolution of the signal. By maintaining the time, the inverse synchro-squeezing algorithm can reassemble a certain representation of the original signal [18].

WSST algorithm operates as follows, by using an analytic wavelet, continuous wavelet transform (CWT) of the input signal is obtained in order to extract instantaneous frequency $\omega_{f}$. A phase transform $\omega_{f}$ is defined, which is proportional to the first derivative of the CWT

$$
\omega_{f}=\frac{\partial t W_{S}(a, b)}{2 \pi j W_{f}(a, b)}
$$

Scales are defined as the ratio of the peak frequency $f_{x}$ to frequency $f$.

$$
a=\frac{f_{x}}{f}
$$

Squeezing the CWT through sections by keeping the phase transform constant, the resulting instantaneous frequency value is reallocated to a particular value at the centroid of the CWT time-frequency region [17].

Entropy is frequently used with the aim of complexity detection in signal processing and defined as, possibility of grey level or $r_{k}$ intensity occurrence in a digital image

$$
p_{r}\left(r_{k}\right)=\frac{n_{k}}{M N} \quad k=0,1,2 \ldots \ldots, L-1
$$

where $M N$ represents the total number of pixels in the image, $n_{k}$ shows the number of pixels holding $r_{k}$ intensity and $\mathrm{L}$ refers to possible intensity level. $p_{r}\left(r_{k}\right)$ vs $r_{k}$ change shows histogram graph.

By using $p_{r}\left(r_{k}\right)$ possibilities, entropy is calculated as,

$$
\widetilde{H}=-\sum_{k=0}^{L-1} p_{r}\left(r_{k}\right) \log _{2} p_{r}\left(r_{k}\right)
$$

It is represented in log base 2 since average information included is in terms of bits.

WSSTs of less noisy and noisy brake are considered as an image matrix and entropy values of every braking interval is computed. As expected, greater noise (noisy brake) resulted in greater entropy value.

\section{Experimental Analysis and Results}

In this study, sound signals acquired from a less noisy and a noisy air disc brake (reported by drivers with unexpected noise issue) which are produced by Ege Fren are investigated. During the experiment, the vehicles with noisy brakes are placed in a silent and vibration free lab environment and sound data have been collected when the driver hit the brake. The force produced by drivers' hit on the pedal is transmitted to brake discs pneumatically as mentioned in the previous section, which ensures that all components of the disc brake subject to are active during data acquisition. 150 seconds audio recordings of two of the brakes was taken by using two Norsonic Type 1228 microphones placed on the brakes with imc CRONOSflex (4 channel sound measurement module) data acquisition unit at a sampling rate of $20 \mathrm{kHz}, 16$-bit resolution and these data are analyzed in MATLAB software. Every braking instant has been detected for both types. The duration between hitting moment and the following 2 seconds is named as braking interval. The analysis has been carried out in the range of $140 \mathrm{~Hz}-5 \mathrm{kHz}$. Figure $2 \mathrm{a}$ shows a sample braking interval of less noisy brake in time domain and related ridges whereas Figure $2 \mathrm{~b}$ shows the wavelet Synchrosqueezed transform of same braking interval.

Duration of every braking is 2 seconds as mentioned before, in the first second the unexpected noise is barely observed whereas the air evacuation of disc brake is particularly dominant in the second half of the braking interval. In contrast, as can be seen in Figure $3 \mathrm{a}$. and $3 \mathrm{~b}$. noisy brake has a stronger ridge and WSST characteristic where the red rectangles indicate the difference clearly in Figure $2 b$ and $3 b$, respectively. No significant difference has been seen in terms of air evacuation between two fault types. In the graphs, while the blue color indicating that there is no wavelet peak or 
signal energy, other colors indicates the wavelet peaks or ridges.

Observations might be made from WSST graphics; however, entropy gives us an objective criterion to measure disorder [19]. Figure 4 represents the entropy of less noisy and noisy brake for every braking interval respectively. The average entropy values for the less noisy and noisy brakes are calculated as $0.65 \pm 0.05 \%(n=39)$ and $0.98 \pm 0.03 \%(n=30)$, respectively. Standard deviations of 7.7 and $3.0 \%$ for the less noisy and noisy brakes, implies that entropy can be concluded as a reliable feature. Numerical variation between the entropy values of the investigated brakes is significantly different from each other and this difference is enough to discriminate the faulty and nonfaulty forms. The scalogram matrix and the quantitative entropy measurements verifies each other bilaterally.
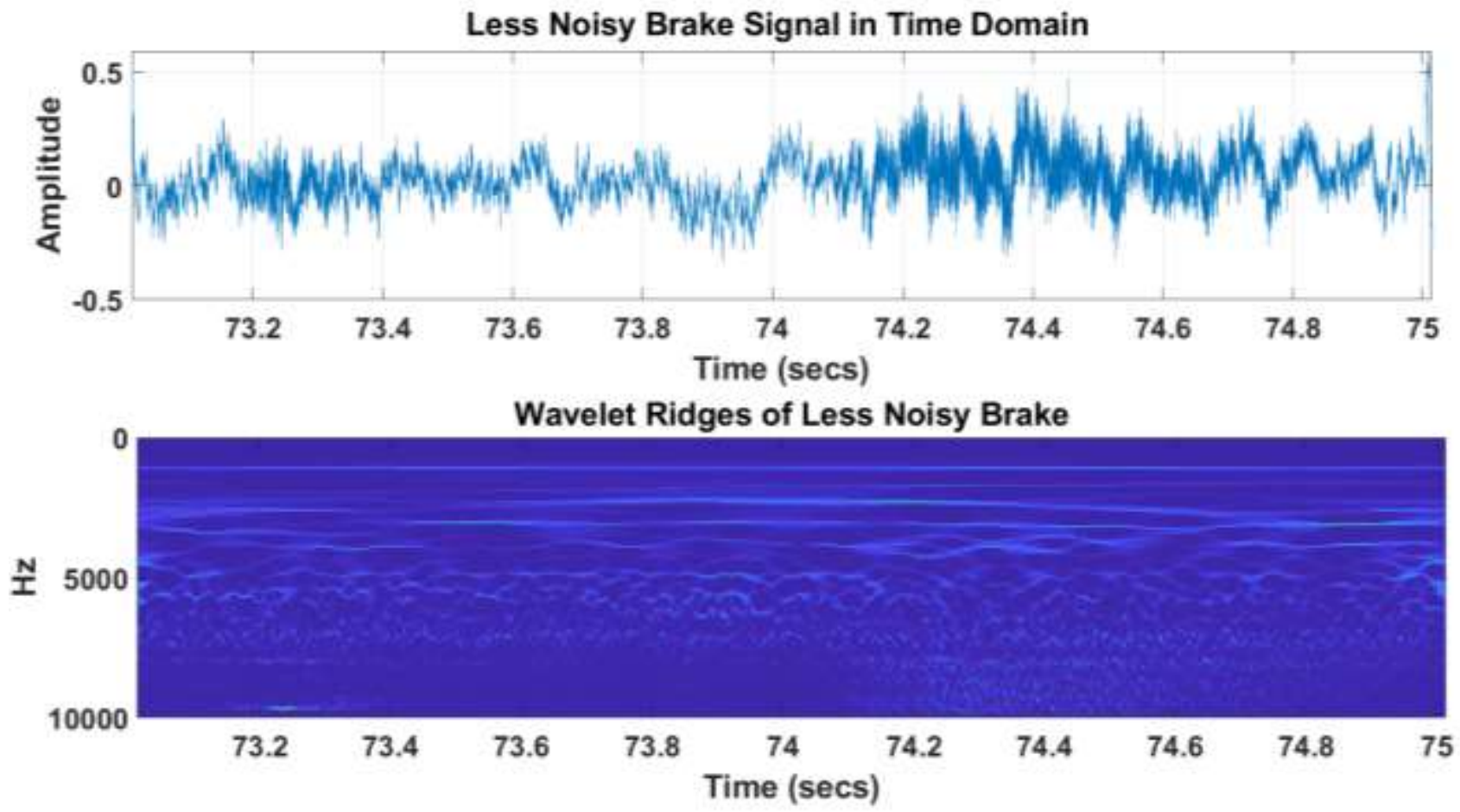

Figure 2a. Time domain representation and wavelet ridges of less noisy brake respectively.

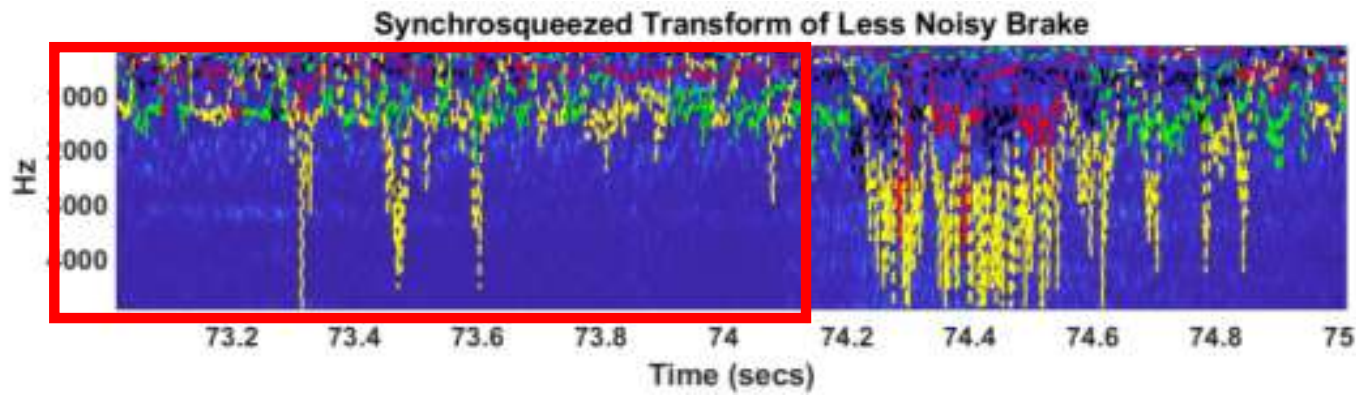

Figure 2b. Wavelet Synchrosqueezed Transform of less noisy brake respectively. 

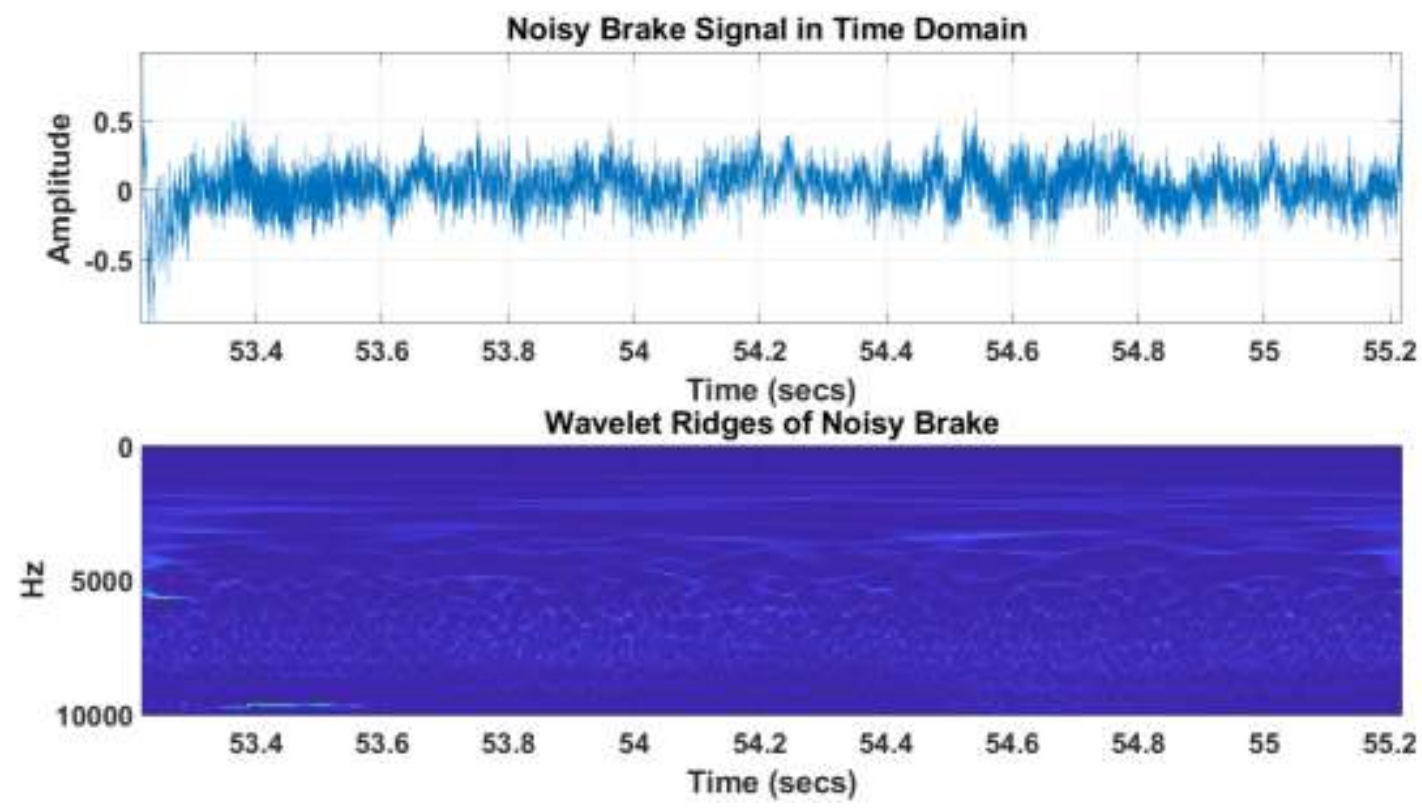

Figure 3a. Time domain representation and wavelet ridges of less noisy brake.

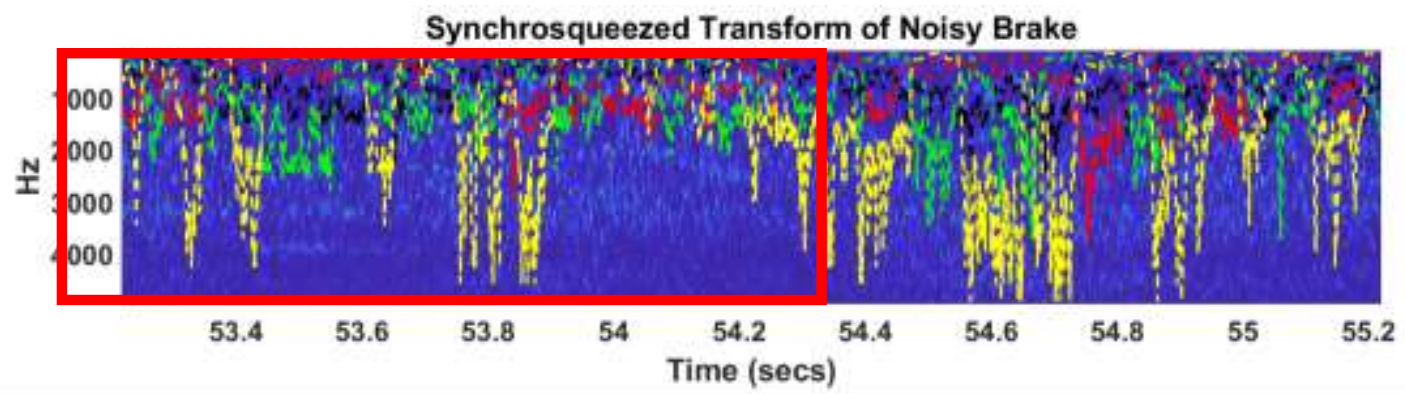

Figure 3b. Wavelet Synchrosqueezed Transform of less noisy brake respectively.

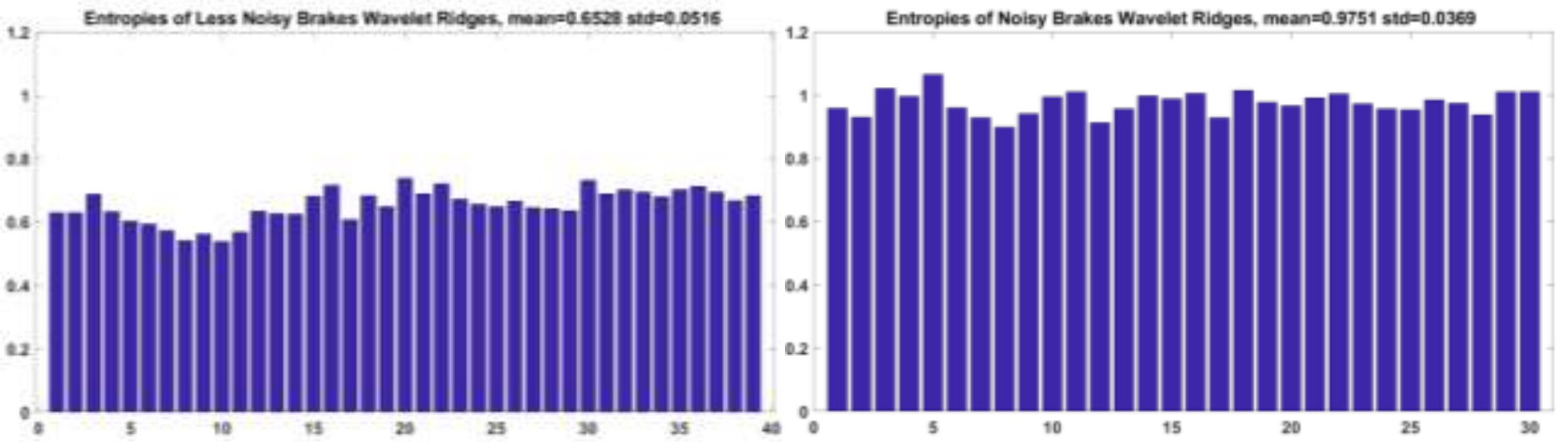

Figure 4. Entropies of Less Noisy brake and Noisy brake for every braking interval, respectively.

\section{Discussion and Future Work}

Noise data acquired from faulty air disc brakes are examined using WSST in this study. It has been shown that WSST shows the differences between these two faults particularly better than frequency analysis only [20]. As a criteria of complexity, average entropy values of two recordings exhibited a considerable distinction. Hence, WSST is assumed to be a promising technique while evaluating fault diagnosis in air disc brakes. Our works on detecting faulty brakes by using signal processing techniques are still ongoing. Qualitative and quantitative features other than entropy will be investigated in detail to classify brakes semiautomatically or automatically. By this means, it will be possible to examine brakes on the vehicle, without removing and reassembling; so that without money and time loss. 


\section{Acknowledgement}

We would like to express our profound appreciation to Ege Fren A.Ş. for providing data and lab environment for data acquisition.

\section{Ethics}

There are no ethical issues after the publication of this manuscript.

\section{References}

1. Beloiu, DM, Ibrahim, RA. 2006. Analytical and experimental investigations of disc brake noise using the frequency-time domain, Structural Control and Health Monitoring. The Official Journal of the International Association for Structural Control and Monitoring and of the European Association for the Control of Structures; 13(1): 277-300.

2. Rhee, SK, Tsang, PHS, Wang, YS. 1989. Friction-induced noise and vibration of disc brakes. Wear; 133(1): 39-45.

3. Chen, X., \& Feng, Z. (2016, June). Time-frequency demodulation analysis for gearbox fault diagnosis under nonstationary conditions. In 2016 IEEE International Conference on Prognostics and Health Management (ICPHM) (pp. 1-6). IEEE.

4. He, D, Cao, H, Wang, S, Chen, X. 2019. Time-reassigned synchrosqueezing transform: The algorithm and its applications in mechanical signal processing. Mechanical Systems and Signal Processing; 117: 255-279.

5. Chen, X, Feng, Z. 2016. Application of reassigned wavelet scalogram in wind turbine planetary gearbox fault diagnosis under nonstationary conditions. Shock and Vibration.

6. Li, H, Xu, F, Liu, H, Zhang, X. 2015. Incipient fault information determination for rolling element bearing based on synchronous averaging reassigned wavelet scalogram. Measurement; 65: 1-10.

7. Peng, Z, Meng, K, G, Chu, FL. 2011. Improved wavelet reassigned scalograms and application for modal parameter estimation. Shock and Vibration; 18(1-2), 299-316.

8. Peng, Z, Chu, F, He, Y. 2002. Vibration signal analysis and feature extraction based on reassigned wavelet scalogram. Journal of Sound and Vibration; 253(5): 1087-1100.
9. Liu, W, Cao, S, Wang, Z, Jiang, K, Zhang, Q, Chen, Y. 2018. A Novel Approach for Seismic Time-Frequency Analysis Based on High-Order Synchrosqueezing Transform. IEEE Geoscience and Remote Sensing Letters; 99: 1-5.

10. Herrera, R. H, Tary, J.B, van der Baan, M, Eaton, DW. 2015. Body wave separation in the time-frequency domain. IEEE Geoscience and Remote Sensing Letters; 12(2): 364-368.

11. Li, D, Liu, H, Gui, $X$, Zhang, X. 2016. An efficient ISAR imaging method for maneuvering target based on synchrosqueezing transform. IEEE Antennas and Wireless Propagation Letters; 15: 1317-1320.

12. Clausel, M, Oberlin, T, Perrier, V. 2015. The monogenic synchrosqueezed wavelet transform: a tool for the decomposition/demodulation of AM-FM images. Applied and Computational Harmonic Analysis; 39(3): 450-486.

13. Ertekin, Z, Özkurt, N, \& Y1lmaz, C. 2017. Disk Fren Sistemlerinde Dalgacık Tepeleri Yöntemi ile Ses Analizi. Cukurova Üniversitesi Mühendislik-Mimarlık Fakültesi Dergisi; 32(4): 193-200.

14. Hwang, W, Han, K, Huh, K. 2012. Fault detection and diagnosis of the electromechanical brake based on observer and parity space. International Journal of Automotive Technology; 13(5): 845851

15. Kampanalı Fren Sistemi Parçaları ve Çalıșması https://otomobilteknoloji.blogspot.com.tr/2016/ 08/kampanali-frensistemi-parcalaricalismasi.html/ (accessed at 31.08.2016)

16. The illustrated wavelet transform handbook: introductory theory and applications in science, engineering, medicine and finance. CRC press. Addison, P. S. 2017

17. Daubechies, I, Lu, J, Wu, HT. 2011. Synchrosqueezed wavelet transforms. An empirical mode decomposition-like tool. Applied and computational harmonic analysis; 30(2): 243-261.

18. https://www.mathworks.com/help/wavelet/gs/waveletsynchrosqueezing.html

19. Gonzalez, R. C, Woods, RE, Eddins, S. L. (2004). Digital image processing using MATLAB (Vol. 624). Upper Saddle River: PearsonPrentice-Hall.

20. Ertekin, Z, Özkurt, N, Yilmaz, C. 2018. In 2018 26th Signal Processing and Communications Applications Conference, İzmir, Turkey, 2018, pp 1-4. 